\title{
Editorial for the Special Issue "Frontiers in Spectral Imaging and 3D Technologies for Geospatial Solutions"
}

\author{
Eija Honkavaara ${ }^{1}\left(\right.$, ${ }^{\text {Konstantinos Karantzalos }}{ }^{2}$, Xinlian Liang ${ }^{1}{ }^{\circledR}$, Erica Nocerino ${ }^{3,4}$, \\ Ilkka Pölönen ${ }^{5}$ (D) and Petri Rönnholm ${ }^{6, *}$ \\ 1 Department of Remote Sensing and Photogrammetry, Finnish Geospatial Research Institute in National \\ Land Survey of Finland, Geodeetinrinne 2, 02431 Masala, Finland \\ 2 Remote Sensing Laboratory, National Technical University of Athens, 15780 Zographos, Greece \\ 3 Aix-Marseille Université, CNRS, ENSAM, Université De Toulon, Bâtiment Polytech, \\ Avenue Escadrille Normandie-Niemen, 13397 Marseille, France \\ 4 Institute of Theoretical Physics, ETH Zurich, 8093 Zurich, Switzerland \\ 5 Faculty of Information Technology, University of Jyväskylä, Mattilanniemi 2, 40100 Jyväskylä, Finland \\ 6 Department of Built Environment, Aalto University, P.O. Box 14100, FI-00076 AALTO, Finland \\ * Correspondence: petri.ronnholm@aalto.fi
}

Received: 11 July 2019; Accepted: 15 July 2019; Published: 19 July 2019

check for updates

\begin{abstract}
This Special Issue hosts papers on the integrated use of spectral imaging and 3D technologies in remote sensing, including novel sensors, evolving machine learning technologies for data analysis, and the utilization of these technologies in a variety of geospatial applications. The presented results showed improved results when multimodal data was used in object analysis.
\end{abstract}

Keywords: hyperspectral imaging; point cloud; sensor integration; data fusion; machine learning; deep learning; classification; estimation; semantic segmentation; object detection; point cloud filtering

\section{Introduction}

Spectral imaging and 3D sensor technologies have developed hugely in recent years, and they can be utilized in a variety of geospatial applications. New cutting-edge hardware and software solutions are emerging, and there is a great need to improve the accuracy, automation level and speed of remote sensing analytics to develop operational geospatial solutions.

The purpose of this Special Issue is to promote the new developments in the field of the combined use of spectral and 3D remote sensing technologies, comprising sensing technologies, thematic information extraction, and geospatial solutions.

\section{Overview of Contributions}

The Special Issue includes papers focusing on forest areas [1-3], agriculture [4], semantic segmentation [5], laser scanning point cloud filtering [6], and detecting moving objects [7]. These interesting approaches applied various sensing technologies, while most of them utilized spectral information.

Tuominen et al. [1] presented a method to classify tree species in a species-rich area with 26 different tree species in total. Hyperspectral data provides interesting possibilities to make reliable and accurate classification. The use of unmanned aerial vehicles (UAVs) for data acquisition is flexible and inexpensive for getting an aerial perspective. Hyperspectral data acquisition was carried out with two new prototypes of a tunable Fabry-Pérot interferometer camera. A rigorous workflow for calibrating hyperspectral data was introduced, and the results showed that accurate radiometric calibration 
improved the performance of classifying algorithms in comparison to the situation when uncalibrated data were used. As a result, it was pointed out that the best classification result was achieved when feature vectors included visible to near infrared (VNIR) and shortwave infrared (SWIR) hyperspectral spectral bands together with many measures of 3D features.

Saarinen et al. [2] utilized VNIR hyperspectral data together with photogrammetric point clouds collected with a UAV. This article took account of the wider aspect of boreal forest biodiversity in addition to tree species classification. The methods presented can be applied as supplementary data or may even partially replace current fieldwork for forest biodiversity assessment. From hyperspectral and 3D geometry, certain biodiversity indicators such as the stand successional stage based on the amount of old deciduous trees, structural diversity, and the amount of dead wood were extracted. As a result, it was found that the structural diversity of a plot was predicted reliably. However, other indicators were not as accurate as the structural diversity.

Zhang et al. [3] proposed a segment-based method for stem detection at the plot level by utilizing terrestrial laser scanning (TLS) data. Detecting stems from TLS point clouds has recently attracted a great deal of attention. In this paper, ground points were filtered out, and the points from branches and foliage were thinned according to curvature values before performing connected component segmentation and the identification of stems. The performance of the method was quite good in the case of trees with simple shape. However, the limits of the method become visible in the cases where trees are characterized by a complex geometry. For the most difficult case, in which data are collected from a single scan and the tree shape is difficult, clear challenges were faced.

Näsi et al. [4] estimated crop biomass and nitrogen content from VNIR hyperspectral and RGB images and 3D photogrammetric point clouds captured with a UAV and a small manned aircraft. These variables are highly important in order to optimize fertilization and to develop economically and environmentally sustainable agricultural practices. Also, in this study, the best results were achieved when calibrated spectral data were utilized together with 3D features.

Because of innovations in deep learning, neural networks have lately emerged in semantic segmentation methods. Papadomanolaki et al. [5] presented an object-based deep learning based semantic segmentation, which was an extension to fully convolutional deep networks. This method was compared with mainstream convolutional and fully convolutional deep networks. Using object-based constraints increased training time moderately. However, the results were equally good, and in some cases slightly better, than with the mainstream methods.

Cai et al. [6] focused on finding ground points from airborne laser scanning data and UAV laser scanning data. This topic has been of great interest for a long time, and many methods exist for this task. The proposed workflow is in line with state-of-the-art approaches that select seed points and detect digital terrain points. In this paper, each step was slightly improved, leading to very good results, comparable to the most accurate methods currently available. It appeared that the resolution of the cloth simulation had a significant impact on the results.

Kandylakis et al. [7] utilized the multi-sensor fusion of hyperspectral, thermal and shortwave infra-red video sensors for near real-time object detection and tracking tasks in challenging visibility conditions. Such methods are crucial for surveillance tasks. This work required both system and software development. Deep learning methods were applied for object detection. Tests in indoor and outdoor environments showed promising results. However, small targets of just a few pixels were not detected.

In [2,5], ISPRS benchmark data sets were utilized, which emphasizes the importance of such data as the results can be conveniently compared among different studies.

\section{Conclusions}

This Special Issue demonstrates and promotes the application of new, powerful spectrometric and 3D sensing technologies and shows the importance of multimodal data integration in a variety of geospatial interpretation tasks. The results showed that the integration of 3D and spectral data 
provided the best results and, moreover, that spectral calibration is also an essential step for improving the performance of the developed approaches.

Acknowledgments: The Guest Editors of this Special Issue would like to thank all authors who have contributed to this volume for sharing their scientific results and for their excellent collaboration. Special thanks are due to the community of distinguished reviewers for their valuable and insightful inputs. The Remote Sensing editorial team is gratefully acknowledged for its support during all phases of the endeavor to successfully complete this volume. In addition, the involvement of ISPRS working groups WG III/4, WG III/1, WG I/5, WG I/1, and WG II/7 is highly appreciated.

Conflicts of Interest: The author declares no conflict of interest.

\section{References}

1. Tuominen, S.; Näsi, R.; Honkavaara, E.; Balazs, A.; Hakala, T.; Viljanen, N.; Pölönen, I.; Saari, H.; Ojanen, H. Assessment of Classifiers and Remote Sensing Features of Hyperspectral Imagery and Stereo-Photogrammetric Point Clouds for Recognition of Tree Species in a Forest Area of High Species Diversity. Remote Sens. 2018, 10, 714. [CrossRef]

2. Saarinen, N.; Vastaranta, M.; Näsi, R.; Rosnell, T.; Hakala, T.; Honkavaara, E.; Wilder, M.A.; Luoma, V.; Tommaselli, A.M.G.; Imai, N.N.; et al. Assessing Biodiversity in Boreal Forests with UAV-Based Photogrammetric Point Clouds and Hyperspectral Imaging. Remote Sens. 2018, 10, 338. [CrossRef]

3. Zhang, W.; Wan, P.; Wang, T.; Cai, S.; Chen, Y.; Jon, X.; Yan, G.A. Novel Approach for the Detection of Standing Tree Stems from Plot-Level Terrestrial Laser Scanning Data. Remote Sens. 2019, 11, 211. [CrossRef]

4. Näsi, R.; Viljanen, N.; Kaivosoja, J.; Alhonoja, K.; Hakala, T.; Markelin, L.; Honkavaara, E. Estimating Biomass and Nitrogen Amount of Barley and Grass Using UAV and Aircraft Based Spectral and Photogrammetric 3D Features. Imaging. Remote Sens. 2018, 10, 1082. [CrossRef]

5. Papadomanolaki, M.; Vakalopoulou, M.; Karantzalos, K.A. Novel Object-Based Deep Learning Framework for Semantic Segmentation of Very High-Resolution Remote Sensing Data: Comparison with Convolutional and Fully Convolutional Networks. Remote Sens. 2019, 11, 684. [CrossRef]

6. Cai, S.; Zhang, W.; Liang, X.; Wan, P.; Qi, J.; Yan, G.; Shao, J. Filtering Airborne LiDAR Data Through Complementary Cloth Simulation and Progressive TIN Densification Filters. Remote Sens. 2019, 11, 1037. [CrossRef]

7. Kandylakis, Z.; Vasili, K.; Karantzalos, K. Fusing Multimodal Video Data for Detecting Moving Objects/Targets in Challenging Indoor and Outdoor Scenes. Remote Sens. 2019, 11, 446. [CrossRef] 